\title{
High-Resolution Infrared Far-IR Spectroscopy from SOFIA, 2005-2025
}

\author{
J. A. Davidson \\ USRA, Moffett Field, CA 94035-1000, USA \\ E. F. Erickson
}

NASA Ames Research Center, Moffett Field, CA 94035-1000, USA

\begin{abstract}
NASA and the DLR are developing SOFIA, the Stratospheric Observatory for Infrared Astronomy: a $2.5 \mathrm{~m}$ telescope in a Boeing 747SP aircraft. By flying in the lower stratosphere, SOFIA will allow astronomical measurements covering the wavelength range from $0.3 \mu \mathrm{m}$ to $1.6 \mathrm{~mm}$, with an emphasis on the spectral regions inaccessible from the ground, particularly the 6-8 $\mu \mathrm{m}$ and $30-300 \mu \mathrm{m}$ regions. SOFIA will see "first light" in 2004; the science program will start in 2005. An operational lifetime of 20 years is planned.

SOFIA will support a diverse and evolving complement of state-of-theart science instruments. Its spectrometers will have resolutions ranging from $\sim 10^{8}$ down to $\sim 100$, as needed to measure absorption and emission from astrophysically significant atoms, ions, molecules, aerosols, and solids. This paper describes the spectrometers being developed to fly on SOFIA soon after first light, and summarizes some of the high-spectral resolution investigations expected.
\end{abstract}

\section{SOFIA and Other FIR/submm Missions}

The 3-m class telescopes on SOFIA and Herschel will improve previously available angular resolution at far-infrared wavelengths by a factor $\sim 3$. SOFIA will fly over 100 flights per year during most of its 20 year lifetime, enabling observations of most IR/submm wavelengths obscured from the ground. The long lifetime and frequent flight opportunities will allow SOFIA to develop and maintain an unmatched variety of sophisticated IR/submm instruments. Thus SOFIA is assured a major role in future high-resolution spectroscopy at these wavelengths.

Ten spectrometers are anticipated for future far-IR and submm ("FIR") space and suborbital missions: SIRTF, SOFIA, Astro-F, Herschel, and JWST. Of the 10 planned spectrometers, five are being developed for SOFIA, and all of these by Principal Investigators (PIs). These spectrometers together cover a wider range of wavelengths than those of any other mission. Briefly these are

EXES (PI John Lacy, University of Texas): The Echelon Cross Echelle Spectrograph is a $5-28.5 \mu \mathrm{m}$ high resolution spectrograph with a maximum resolution $\sim 3 \mathrm{~km} \mathrm{~s}^{-1}$. 
SAFIRE (PI Harvey Moseley, NASA-GSFC): The Submillimeter And Far-InfraRed Experiment is a versatile imaging Fabry-Perot spectrograph working in the range of 100 to $655 \mu \mathrm{m}$.

GREAT (PI Rolf Guesten, MPIfR): The German REceiver for Astronomy at $\mathrm{THz}$ frequencies is a dual channel heterodyne instrument with high spectral resolution in the 63 to $188 \mu \mathrm{m}(1.6$ to $4.7 \mathrm{THz})$ region.

CASIMIR (PI Jonas Zmuidzinas, Caltech): The Caltech Airborne Submillimeter Interstellar Medium Investigations Receiver is a FIR heterodyne spectrometer, working in the wavelength range from 150 to $600 \mu \mathrm{m}$ $(500-2000 \mathrm{GHz})$ with spectral resolution of $>0.3 \mathrm{~km} \mathrm{~s}^{-1}$.

FIFI-LS (PI Albrecht Poglitsch, MPE Garching): The Far-Infrared Field Imaging Line Spectrometer is a German integral-field unit spectrometer with spectral resolution $150-300 \mathrm{~km} \mathrm{~s}^{-1}$. It will operate in the 42 to $210 \mu \mathrm{m}$ region with two wavelength channels and two separate array detectors.

Future spectrometers on SOFIA can be anticipated by looking at Figure 1. Clearly there will be a need for for a high resolution instrument(s) to provide contiguous wavelength coverage from $\sim 20-200 \mu \mathrm{m}$, for example.

\section{FIR High-Resolution Spectroscopy}

A wealth of important scientific topics remains to be explored with FIR spectroscopy. "High resolution" is a relative term, which we interpret to mean both higher resolution than has previously been utilized, and resolution sufficient to resolve the spectral features of astronomical sources. High resolution increases sensitivity for line measurements on sources with bright continuum emission, as does the improved angular resolution provided by the SOFIA telescope for extended sources.

Important FIR spectral diagnostics arise in a wide variety of sources, including AGNs, HII regions, planetary nebulae, photodissociation regions (PDRs), circumstellar envelopes, and molecular clouds. The FIR wavelengths suffer little extinction, and so allow observation of phenomena in highly obscured regions such as our Galactic Center. Fine structure lines arising from low level transitions in atoms and ions characterize the originating gas from cool to hot. For example, [C II] at $158 \mu \mathrm{m}$ is a major coolant of PDRs, which are principal constituents of the ISM. The Ne VI line at $24.3 \mu \mathrm{m}$ is a signature of hot gas in AGNs. A wide variety of rotational molecular lines, including high-J $\mathrm{CO}, \mathrm{H}_{2} \mathrm{O}, \mathrm{H}_{2}, \mathrm{HD}$, $\mathrm{OH}$ and other hydrides extend throughout the far-IR and submm regions. These many FIR lines enable observations to probe the pressure, density, luminosity, excitation, mass distribution, chemical composition, heating and cooling rates, and kinematics in the various components of the ISM. We give a few examples of the numerous phenomena characterized by these lines:

Extragalactic Star Formation: How do interactions between high mass stars and the ambient medium relate to the star formation history? The ISM in irregulars and spirals can differ greatly: SN rate, stellar winds, density, IMF. Lines of [O III] $52 \mu \mathrm{m}$ and [O I] $63 \mu \mathrm{m}$, with widths $\sim 30 \mathrm{~km} \mathrm{~s}^{-1}$, probe the 


\section{Anticipated Spectroscopic Capabilities, 2003-2025}

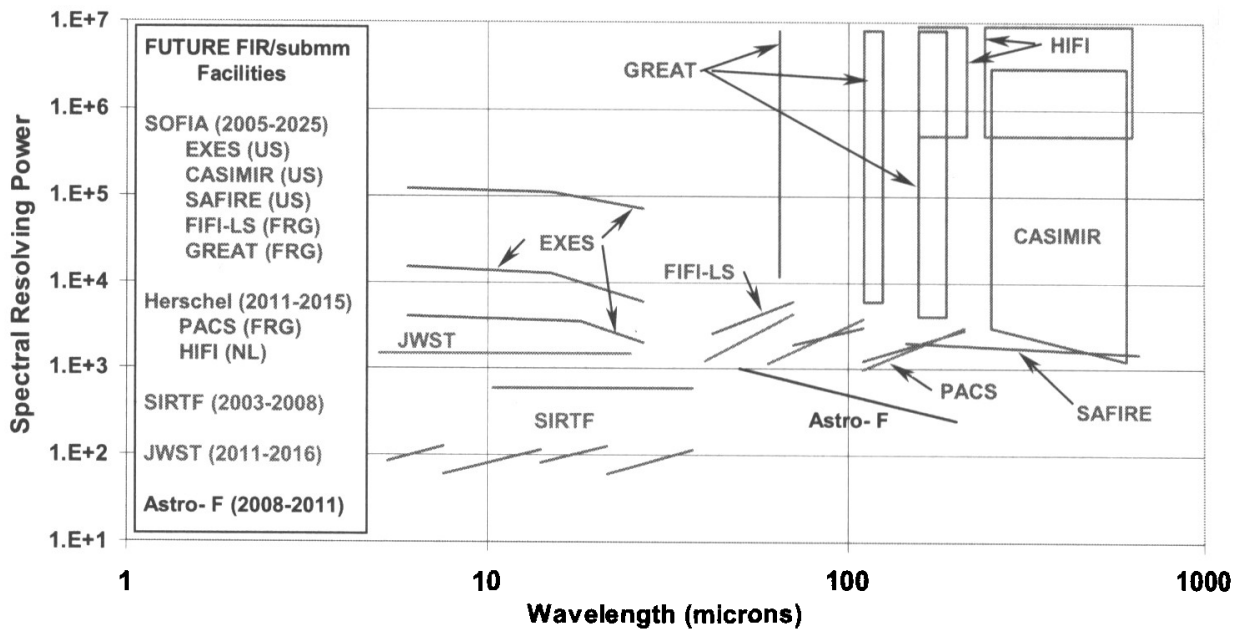

Figure 1. Future spectrometers on SOFIA

content and dynamics of photoionized and photodissociated gas. The [O III] 52 $\& 88 \mu \mathrm{m}$ and [S III] $33 \mu \mathrm{m}$ lines yield the density and excitation of ionized gas.

Star formation in the Milky Way: How do the details of infall, accretion, and outflow in low mass, young stellar objects affect the resulting stellar properties? Narrow high-J CO and [O I] $63 \mu \mathrm{m}$ lines originating near objects in Taurus should yield a direct measure of the mass infall rate. Material accreting onto pre- and young stellar disks will produce $\mathrm{OH}$ doublets at 84 and $119 \mu \mathrm{m}$ that are sensitive to the preshock density and the shock velocity, which together determine the accretion rate. The morphology and mass loss rate of outflows will be revealed by [O I] 63 and $145 \mu \mathrm{m}$ lines produced as the natal material is shocked by the outflowing gas.

Mass distribution in cirumstellar disks around visible T-Tauri stars: Planets forming in these disks should cause disk clearing, which will be reflected in line profiles of $\mathrm{H}_{2}$ at 28,17 , and $12 \mu \mathrm{m}$, as well as lines of methane and water. These observations require spectral resolutions of a few $\mathrm{km} \mathrm{s}^{-1}$.

Interstellar chemistry: What is the relative importance of gas-phase chemistry versus dust-grain surface chemistry in dark clouds? Depletion of molecules onto dust grains in dark clouds would explain high deuterium fractionation. The abundance of $\mathrm{H}_{2} \mathrm{D}^{+}$is the key to understanding this process. The lowest energy-level transition $(101 \rightarrow 000)$ at $219 \mu \mathrm{m}(1.37 \mathrm{THz})$ should be detectable with SOFIA's heterodyne spectrometers. 


\section{Spectroscopic Observing Opportunities with SOFIA}

Development and maintenance of the first generation SOFIA spectrometers described above will be the responsibility of the PIs. General Investigators (GIs) will be allowed to propose observations with a PI's instrument in collaboration with him. In the future, facility spectrometers may be available for GI investigations without the need for collaboration with a PI. Performance summaries for all the first generation SOFIA instruments are currently available at http://sofia.arc.nasa.gov/Science/instruments/sci_instruments.html.

SOFIA is a joint program of NASA in the U.S. and DLR in Germany. Observing time will be awarded by annual peer review of proposals, with roughly $80 \%$ of the time granted by the U.S. and $20 \%$ of the time granted by Germany. International proposals may be submitted to either time allocation committee. Observing opportunities for General Investigators are expected to begin in 2006.

\section{Conclusions}

The interstellar media in galaxies are complex and fundamental components of the universe. Many physical and chemical processes - such as star and planetary system formation and the evolution of elemental abundances through stellar life cycles - are yet to be understood.

Far-IR and submm lines bring a wealth of knowledge about the ISM and the processes hidden therein. These lines require measurements with high spectral and spatial resolution from space or suborbital platforms. SOFIA and Herschel will improve FIR angular resolution by a factor $\sim 3$ relative to past FIR telescopes, and will provide many of the needed high spectral resolution capabilities. SOFIA's long lifetime will encourage future spectrometer development, to extend even further the Frontiers of High Resolution Spectroscopy. 